Research Paper

\title{
Qualitative Alpha-defensin Versus The Main Available Tests For The Diagnosis Of Periprosthetic Joint Infection: Best Predictor Test?
}

\author{
Giovanni Riccio ${ }^{1}$, Luca Cavagnaro², Wassim Akkouche³, Giuliana Carrega', Lamberto Felli², Giorgio \\ Burastero ${ }^{\natural}$ \\ 1. Centro MIOS, S.C. Malattie Infettive, ASL 2 Savonese Ospedale di Pietra Ligure-Albenga \\ 2. Clinica Ortopedica - Ospedale Policlinico San Martino, Genova, Italy \\ 3. Patologia Clinica e Microbiologia, ASL 2 Savonese, Liguria \\ 4. Centro MIOS, S.C. Ortopedia 2, ASL 2 Savonese Ospedale di Pietra Ligure-Albenga \\ $\triangle$ Corresponding author: Wassim Akkouche, akkouche@gmail.com \\ ( $)$ Ivyspring International Publisher. This is an open access article distributed under the terms of the Creative Commons Attribution (CC BY-NC) license \\ (https://creativecommons.org/licenses/by-nc/4.0/). See http://ivyspring.com/terms for full terms and conditions.
}

Received: 2018.03.31; Accepted: 2018.07.05; Published: 2018.07.27

\begin{abstract}
Introduction: Biomarkers such as $\alpha$-defensin demonstrated to be a potentially useful option in periprosthetic joint infection (PII) diagnosis. Recently, a new point-of-care test for $\alpha$-defensin level detection in synovial fluid has been commercialized in Europe. The aim of this study is to compare the $\alpha$-defensin test (Synovasure ${ }^{\mathrm{TM}}$ ) diagnostic ability with the main available clinical tests for periprosthetic joint infection diagnosis in a practical clinical setting of a Bone Infection Unit.

Methods: Between 2015 and 2017, 146 patients with suspected chronic PJI were screened with Synovasure $^{\mathrm{TM}}$. Seventy-three of these met the Musculoskeletal Infection Society (MSIS) criteria and were included in the analysis. According to MSIS criteria, 40 patients $(54.7 \%)$ were classified as infected and 33 (45.3\%) as not infected. The results obtained with Synovasure ${ }^{\mathrm{TM}}$ were recorded and compared with standard diagnostic methods for PJI diagnosis.

Results: Synovasure ${ }^{\mathrm{TM}}$ showed a sensitivity of $85.0 \%(95 \% \mathrm{Cl} 70.2$ to 94.3$)$ and a specificity of 96.9 \% (95\% Cl 83.8 to 99.9$)$ for PJI detection. The positive likelihood ratio of Synovasure ${ }^{\mathrm{TM}}$ was 27.2 $(95 \% \mathrm{Cl} 3.9$ to 188.1$)$ and the negative likelihood ratio was $0.2(95 \% \mathrm{Cl} 0.1$ to 0.3$)$. The diagnostic odds ratio was 181.3 (95\% Cl 20.7 to 1590.4$)$. Synovasure ${ }^{T M}$ demonstrated a statistical significant difference when compared to Erythrocyte Sedimentation Rate (ESR) and C-reactive Protein (CRP) specificity (at least one positive test) and preoperative culture sensitivity (1 positive culture).

Conclusion: Our findings show that Synovasure ${ }^{T M}$ sensitivity is lower than quantitative $\alpha-D$ test, but when compared to the main available tests shows a good specificity and the highest DOR. On the SF it is the easier test to do, due to the fact that it needs a minimal amount of SF and it is not limited by blood contamination or antibiotic use. Whereas there is no single standalone test, Synovasure ${ }^{T M}$ should be considered a reliable additional test for periprosthetic joint infection diagnosis in everyday clinical practice.
\end{abstract}

Key words: Alpha-defensin; Synovasure; Periprosthetic joint infection; Synovial fluid

\section{Introduction}

Periprosthetic joint infection (PJI) is considered to be one of the most challenging complications in the orthopaedic field. PJI diagnosis and management remain a source of concern and a major socioeconomic burden worldwide [1,2]. It accounts for $15.6 \%$ of surgical revisions in total hip arthroplasty (THA) and is considered to be the dominant reason for failure during the first 15 years, after primary total knee 
arthroplasty (TKA) [3,4]. Numerous efforts have been made to improve the diagnosis of PJI. In 2011, the Musculoskeletal Infection Society (MSIS) provided a consensus statement in order to accomplish a shared definition of PJI [5]. This algorithm was modified in 2013 [6]. Currently, the diagnosis of PJI relies on clinical parameters, serum biomarker (Erythrocyte Sedimentation Rate (ESR) and C-reactive Protein (CRP)), synovial fluid white blood cell (WBC) count and polymorphonuclear cell percentage, synovial fluid Leukocyte Esterase (LE) test, periprosthetic tissue culture and histological analyses, but none of them have proved to be a reliable test individually.

In the last few years, a lot of attention has been focused on biomarkers in synovial fluid (SF). In 2014, a prospective study including 95 patients with painful hip or knee arthroplasties in which the authors evaluated 16 biomarkers involved in the immune reaction after a PJI. Five of them (a-defensin 1.3, neutrophil elastase 2, bactericidal/permeability increasing protein, neutrophil gelatinase-associated lipocalin and lactoferrin) showed a $100 \%$ sensitivity and specificity in diagnosing PJI [7].

Alpha defensin (a-D) is a natural peptide of the innate immunity released mainly from polymorphonuclear cells in response to pathogens such as Gram-positive and Gram-negative bacteria, fungi and enveloped viruses. The antimicrobial properties of this peptide are due to the ability to induce depolarization of the cell membrane by means of a "channel-like" pore formation [8,9]. Several published reports claimed a-D to be the optimal biomarker for PJI showing high diagnostic accuracy and promising results when compared to other available tests [10-13]. This data was obtained through quantitative testing with enzyme-linked immunosorbent assay (ELISA) after centrifugation of the SF. Recently a new point-of-care test for PJI identification (Synovasure ${ }^{\mathrm{TM}}$, Zimmer Inc., Warsaw; IN) was commercialized in Europe. Synovasure ${ }^{\mathrm{TM}}$ was not cleared or approved by the U.S. Food and Drug Administration but it received the $\mathrm{CE}$ (European Conformity) marking in Europe. It provides a simple "yes or no" answer after 10 minutes. Despite its widespread use to date only a little data about Synovasure ${ }^{\mathrm{TM}}$ accuracy and reliability has been published. Moreover, some recent reports claimed differences in sensitivity and specificity between qualitative and quantitative analysis of a-D $[14,15]$.

We conducted a single-centre retrospective study based on a prospectively collected dataset to (1) assess the sensitivity, specificity and diagnostic ability of the Synovasure ${ }^{\mathrm{TM}}$ and (2) to compare the obtained results with those achieved with other available clinical tests for PJI diagnosis in a practical clinical setting of a regional reference Bone Infection Unit.

\section{Materials and Methods}

From November 2015 to April 2017, a total of 146 patients were enrolled in a PJI study using the Synovasure $^{\mathrm{TM}}$ test. Seventy-three patients did not match the MSIS inclusion criteria, leaving 73 patients for final statistical analysis.

All patients gave written and informed consent to all the diagnostic and surgical procedures. Local Institutional Review Board approval was obtained before the retrospective evaluation.

Inclusion criteria for this investigation were (1) patients with painful arthroplasty under evaluation for possible infection or arthroplasty revision (2) enough clinical and/or laboratory data for patient classification according to MSIS criteria (3) sufficient SF for a-D test.

Antibiotic therapy at the moment of SF aspiration, SF blood contamination and metallosis were not considered exclusion criteria.

Data about age, sex, relevant comorbidities, involved joint, reason for revision, SF blood contamination, antibiotic therapy at the time of SF aspiration and type of surgical treatment were recorded for each patient (Table 1).

According to our diagnostic flow chart, all subjects were screened for serum ESR/CRP and SF aspiration was performed. Serum ESR and CRP were evaluated preoperatively. According to the best available evidence [16], a cut-off level of $30 \mathrm{~mm} / \mathrm{hr}$ for ESR and $10 \mathrm{mg} / \mathrm{L}$ for CRP were chosen for positivity assessment. The test was considered positive if both serum analyses were positive. SF aspiration was always performed by a highly trained infectious disease consultant (more than 20 years' experience in Musculoskeletal infections). A well-trained nurse (more than 10 years of duty in a bone infection unit) assisted all the procedures. SF aspiration was carried out in a dedicated room under sterile conditions (3 times preparation of the skin with alcohol solution and draping) using a 21 Gauge needle. A freehand technique was employed for knee aspirations whereas hip aspirations were performed under fluoroscopy guidance. Immediately after the procedure, SF was partitioned for each laboratory investigation according to the collected volume. A separate sterile tube was obtained for microbiological culture.

Synovasure $^{\mathrm{TM}}$ was performed in the included patients prior to any SF manipulation according to the manufacturer's instructions. The test was performed and read by an experienced operator (more than 20 years-experience in Musculoskeletal infections and more than 1000 joint aspirations performed). The test 
kit comprised: a single use chromatographic device, a premeasured vial of dilution buffer, a disposable Microsafe tube, and a sample cup. The SF is placed in the sample cup. Dilution is obtained by collecting SF with the disposable Microsafe ${ }^{\circledR}$ tube and adding the sample to the premeasured dilution buffer. After gentle hand mixing, 3 drops of the obtained mix is added to the cassette. During its migration through the device the sample mixes with the gold conjugate labelled anti-defensin antibodies. The test was read after 10 minutes incubation. The presence of the control line (marked as $\mathrm{C}$ on the testing cassette) in all performed tests, confirmed the test validity. The test was considered positive if a second line (marked as a-D on the testing cassette) appeared.

LE test was obtained in 67 patients. LE test was accomplished using the Multistix 10 SG (Siemens Healthcare Diagnostics Inc., Tarrytown, New York) reagent strip. The test is a simple colorimetric method and provides a semiquantitative result related to the concentration of leukocyte cells in the SF sample. The method is interpreted as negative (white), trace (off-white),+ (slightly purple), ++ (light purple) or +++ (dark purple). SF was previously centrifuged at $6000 \mathrm{~g}$ for $2 \mathrm{~min}$ if blood contamination was detected. A trained operator evaluated and registered the test strip data. We considered ++ and +++ results as positive tests. [17]
WBC count and characterization were performed using an advanced automated hematological analyzer the Sysmex XN-9000 (Sysmex, Inc. Kobe, Japan) equipped with a specifically designed unit for body fluids analysis (XN-BF), using fluorescent flow cytometry with hydrodynamic focusing. Since the increased viscosity may impair sample aspiration or prevent appropriate mixing a pre-treatment of SF samples with the enzyme hyaluronidase (HY) was made.

SF culture was obtained in all patients. Immediately after joint aspiration, a separate aliquot of SF was inoculated in aerobic (BacT / ALERT FA PLUS) and anaerobic (BacT / ALERT FN PLUS) bottles and taken to the laboratory by the nurse for microbiological testing. The bottles were incubated in the BacT / ALERT 3D instrument (software version B.40 Rel. 4). Gram staining was performed directly from the positive liquid, which was also inoculated in common solid media for subculture. Cultures were incubated for at least 14 days. The retrieved microorganisms along with negative results were recorded.

Sixty-seven out of the 73 enrolled patients underwent surgery. All revisions were performed by the same experienced surgeon. Three to 6 periprosthetic bioptic samples were sent to microbiological analysis by default.

Table 1. Main demographic and surgical data.

\begin{tabular}{cc}
\hline Variable & Data \\
\hline No of patients & 73 \\
\hline Sex & $30 \mathrm{M}$ \\
& $43 \mathrm{~F}$ \\
\hline Mean age (years) & $68.7 \pm 10.5$ \\
& 6 Systemic Inflammatory Disease $(8.2 \%)$ \\
& 7 Cardiopathy $(9.6 \%)$ \\
Relevant Comorbidities & 6 Diabetes mellitus $(8.2 \%)$ \\
& 3 Corticosteroid Therapy $(4.1 \%)$ \\
& 3 Renal failure $(4.1 \%)$ \\
Site & 49 Knees $(67.1 \%)$ \\
& 22 Hips $(30.1 \%)$ \\
& 2 Shoulders $(2.8 \%)$ \\
Reason for Revision & 40 PJI $(54.8 \%)$ \\
& 26 Aseptic loosensing $(35.6 \%)$ \\
& 3 Wear $(4.1 \%)$ \\
& 3 Instability $(4.1 \%)$ \\
& 1 Stiffiness $(1.4 \%)$ \\
SF blood contamination & 49 no $(67.1 \%)$ \\
& 24 yes $(32.9 \%)$ \\
\hline Antibiotic therapy at the time of SF & 64 no $(87.7 \%)$ \\
aspiration & 9 yes $(12.3 \%)$ \\
Surgical treatment & 37 Two-stage $(92.5 \%)$ \\
(Septic revisions) & 2 One-stage $(5 \%)$ \\
& 1 Gilderstone $(2.5 \%)$ \\
\hline
\end{tabular}


According to intraoperative findings, frozen section for definitive histology evaluation was performed. An experienced pathologist specialized in musculoskeletal disorders processed all the specimens and interpreted the obtained results.

\section{Statistical analysis}

Continuous variables were reported as mean \pm standard deviation and compared using the independent Student t-test or Mann-Whitney U test. Categorical variables were expressed as the number of cases or percentage. The sensitivity, specificity, positive likelihood ratio (LR+), negative likelihood ratio (LR-), Diagnostic Odds ratio (DOR) and relative Confidence Intervals (CI) were calculated for $a-D$, serum ESR/CRP, LE test, SF leukocyte count, preoperative culture, frozen section and definitive histology using MSIS criteria as the reference standard. SF polymorphonuclear cell percentage was not included in the analysis because of the low number of screened patients. We did not calculate the positive and negative predictive values and accuracy of the tests because the prevalence of PJI in our cohort of patients did not reflect the prevalence of PJI in the standard population. Sensitivity and specificity of a-D test were compared with other tests using the McNemar's Chi square test. For all the analysed data, a p-value of $<0.05$ was considered statistically significant.

\section{Results}

Of the 73 included patients, 40 were considered septic according to modified MSIS criteria. The septic cohort of patients was composed of 23 males and 17 females with an average age of $68.0 \pm 12.4$ years. All PJI were classified as chronic infections according to Zimmerli classification [18]. According to joint site distribution, there were 19 THA, 19 TKA and 2 reverse shoulder arthroplasties (RSA). Thirty-seven patients underwent a two-stage revision after PJI diagnosis, 2 sustained a one-stage revision. One patient was treated with a Girdlestone resection arthroplasty.

In this group, Synovasure ${ }^{\mathrm{TM}}$ tests were positive in 34 cases and negative in 6 . All the negative patients had negative preoperative cultures, but at least 3 positive intraoperative cultures for the same microorganism. These discordant cases are described below.

The first patient suffered from Rheumatoid Arthritis (RA) and was under immunosuppressant therapy at the moment of evaluation. A THA infection diagnosis with the evidence of a communicating sinus was performed. An LE test was not performed because of blood contamination in the sample, even after centrifugation. Intraoperative frozen section was positive as well as definitive histology. Methicillin-Resistant Staphylococcus aureus (MRSA) was isolated from 4 out of 6 intraoperative cultures. A one-stage procedure was carried out.

The second patient also had a history of RA, treated with steroid and immunosuppressant therapy. All preoperative analyses apart from CRP were negative. Intraoperatively, purulent synovial fluid was observed and a hip spacer was implanted. All the intraoperative cultures were positive (polimicrobic flora). The frozen section and definitive histology were also positive.

The third patient was diagnosed with a THA infection according to intraoperative findings. Streptococcus anginosus was isolated in 3 culture samples. Definitive histology was positive even if the frozen section was not. LE test resulted negative. The only preoperative positive tests were ESR and CPR. According to intraoperative macroscopic analysis, the patients underwent a two-stage revision.

The fourth patient had a history of RA, treated with steroid and immunosuppressant therapy. All preoperative analyses but CRP were negative. Intraoperatively, purulent synovial fluid was observed and a hip spacer was implanted. All the intraoperative cultures were positive (polimicrobic flora). The frozen section and definitive histology were also positive.

The fifth patient was diagnosed with a THA infection after 11 surgical procedures. The only positive preoperative results were ESR and CRP. Intraoperatively, a Girdlestone procedure was performed because of the massive bone loss and the very poor general condition of the patient. Methicillin-Resistant Staphylococcus epidermidis (MRSE) was obtained from 3 intraoperative tissue cultures.

The last false negative patient had a TKA infection with positive synovial WBC count while ESR/CRP and LE tests were negative. Staphylococcus capitis was isolated in 4 intraoperative samples. A two-stage procedure was carried out with positive outcome.

The second group of patients (33 patients) considered aseptic according to the MSIS criteria was composed of 7 males and 26 females with an average age of $69.5 \pm 7.5$ years. Three THA's and 33 TKA's were included. All but 6 patients underwent total joint arthroplasty revision. One patient had a positive preoperative culture, which was considered as a contaminant.

In this group, Synovasure ${ }^{\mathrm{TM}}$ showed one false positive result. This patient underwent evaluation for a painful hip arthroplasty. Preoperative blood tests 


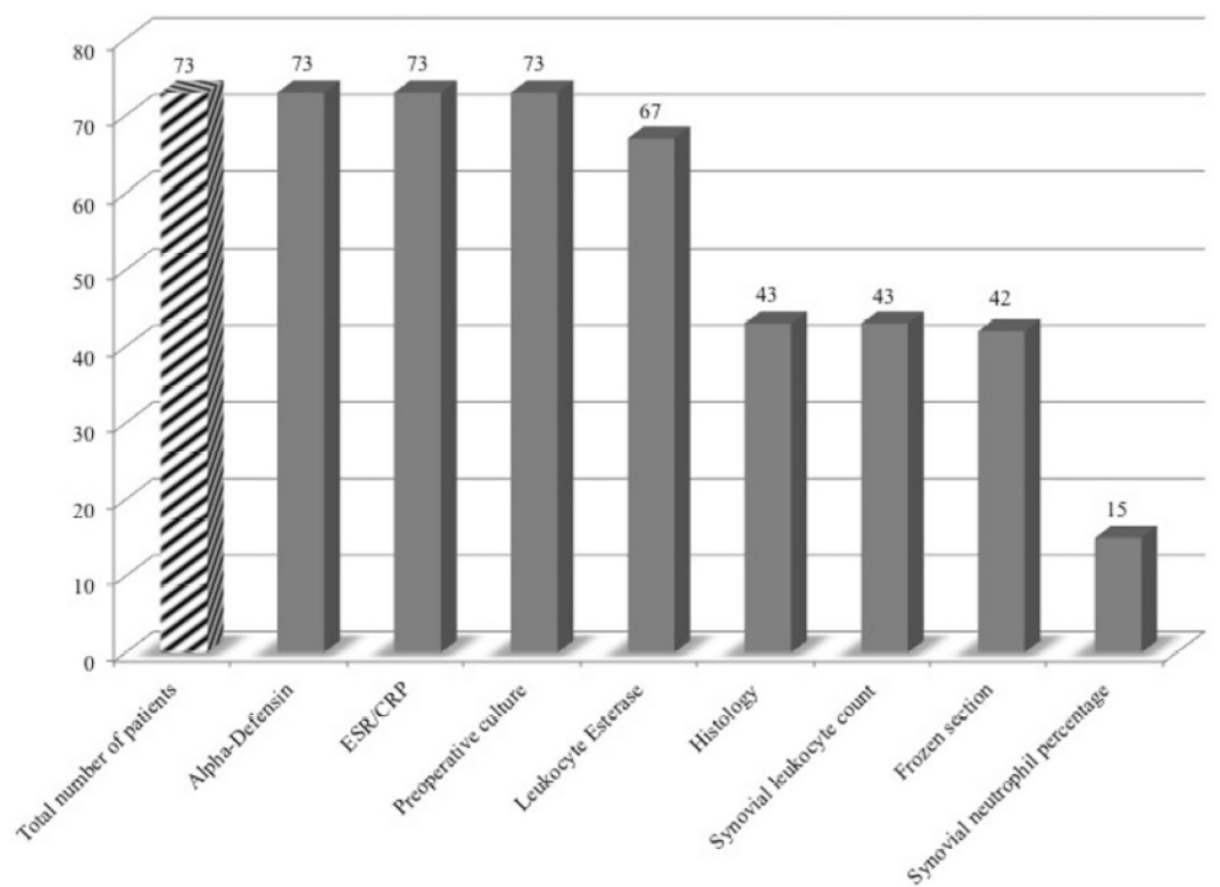

Figure 1. The figure shows the number of patients screened for each test. The striped column represents the total number of patients included in the study.

were mildly positive and purulent SF was obtained even if the LE test and preoperative cultures were negative. Intraoperative findings revealed an intense inflammatory reaction of the periprosthetic tissues without evidence of metallosis but the intraoperative microbiological and histological analysis were negative.

All patients underwent Synovasure ${ }^{\mathrm{TM}}$ testing as well as blood ESR/CRP tests and preoperative culture. An LE test was performed on 67 patients $(91.8 \%)$. In 2 patients The LE test was not provided due to an SF shortage. In 4 patients, the massive blood contamination even after sample centrifugation prevented the test's reliability.

Due to insufficient SF retrieval, a synovial leukocyte count and neutrophil percentage were evaluated in 43 and 15 patients, respectively. Definitive histology and a frozen section were performed on 43 and 42 patients, respectively (Figure $1)$.

Overall, Synovasure ${ }^{\mathrm{TM}}$ showed a sensitivity of $85.0 \%$ (95\% CI 70.2 to 94.3 ) and a specificity of $96.9 \%$ (95\% CI 83.8 to 99.9 ). Synovasure ${ }^{\mathrm{TM}}$ demonstrated a statistical significant difference when compared to ESR/CRP specificity and preoperative culture sensitivity (1 positive culture) (Table 2$)$.

The LR+ of a-D test was 27.2 (95\% CI 3.9 to 188.1) and the LR- was 0.2 (95\% CI 0.1 to 0.3$)$. The DOR was 181.3 (95\% CI 20.7 to 1590.4) (Table 3).

Nine patients were under antibiotic treatment at the moment of $a-D$ testing. The sensitivity and specificity values of a-D test in this specific subset of patients were $85.71 \%$ (95\% CI 42.1 to 99.6 ) and $100.0 \%$ (95\% CI 15.8 to 100.0), respectively.

SF blood contamination was reported in 24 patients. Synovasure ${ }^{\mathrm{TM}}$ showed a sensitivity of $80.0 \%$ (95\% CI 51.9 to 95.7 ) and a specificity of $100.0 \%$ (95\% CI 71.5 to 100.0) in this group, while the same parameters for non-contaminated cohort of patients were $88.0 \%$ (95\% CI 68.8 to 97.4 ) and $95.45 \%$ (95\% CI 77.2 to 99.9$)$.

No statistically significant difference was observed when comparing sensitivity and specificity values between the antibiotic-treated and the antibiotic-free population neither between contaminated blood and non-contaminated SF samples.

\section{Discussion}

The main findings of the presented study are that Synovasure ${ }^{\mathrm{TM}}$ showed a sensitivity of $85.0 \%$, a specificity of $96.9 \%$, a LR+ of 27.2 and a LR- of 0.2 . The DOR was 181.3. When compared to other available tests for PJI diagnosis, Synovasure ${ }^{\mathrm{TM}}$ demonstrated a statistical significant difference when compared to ESR/CRP specificity and preoperative culture sensitivity ( 1 positive culture). Synovasure ${ }^{\mathrm{TM}}$ provided 6 false negatives and 1 false positive results. Among the false negatives, 3 patients were under immunosuppressive therapy. In all cases, PJI diagnosis was achieved with intraoperative analyses. In the false positive patient, an inflammatory reaction of periprosthetic tissues without evidence of metallosis was detected intraoperatively. 
Table 2. Sensitivity and specificity data. The $P$ values showed statistically significant differences only for ESR/CRP specificity and Culture sensitivity (bold type).

\begin{tabular}{ccccc}
\hline & $\begin{array}{c}\text { Sensitivity } \\
(\%)(\mathbf{9 5} \% \mathbf{C I})\end{array}$ & P value & $\begin{array}{c}\text { Specificity } \\
(\%)(\mathbf{9 5} \% \mathbf{C I})\end{array}$ & P value \\
\hline Alpha-Defensin & $85.0(70.2$ to 94.3$)$ & & $96.9(83.8$ to 99.9$)$ & \\
\hline ESR/CRP & $85.7(71.5$ to 94.6$)$ & 1.000 & $66.7(48.2$ to 82$)$ & $\mathbf{0 . 0 0 5}$ \\
$\begin{array}{c}\text { Preoperative } \\
\text { culture }\end{array}$ & $65.0(\mathbf{4 8 . 3}$ to 79.4$)$ & $\mathbf{0 . 0 2 7}$ & $97.0(84.2$ to 99.9$)$ & 1.000 \\
\hline $\begin{array}{c}\text { Leukocyte } \\
\text { Esterase }\end{array}$ & $78.4(61.8$ to 90.2$)$ & 0.221 & $96.7(82.8$ to 99.9$)$ & 1.000 \\
\hline $\begin{array}{c}\text { Leukocyte } \\
\text { count }\end{array}$ & $83.3(62.2$ to 95.3$)$ & 0.683 & $89.5(66.9$ to 98.7$)$ & 1.000 \\
\hline $\begin{array}{c}\text { Frozen section } \\
\text { Histology }\end{array}$ & $79.0(54.4$ to 94.0$)$ & 0.617 & $100.0(85.2$ to 100.0$)$ & 1.000 \\
\hline
\end{tabular}

ESR/CRP: Erythrocyte Sedimentation Rate/C-Reactive Protein

CI: Confidence Interval

Table 3. Diagnostic Odds Ratio (DOR), Positive Likelihood Ratio (LR+) and Negative Likelihood Ratio (LR-) of different tests.

\begin{tabular}{cccc}
\hline & DOR $(\mathbf{9 5} \% \mathbf{C I})$ & LR+ (95\% CI) & LR- (95\% CI) \\
\hline Alpha-Defensin & $181.3(20.7$ to 1590.4$)$ & $27.2(3.9$ to 188.1$)$ & $0.2(0.1$ to 0.3$)$ \\
\hline ESR/CRP & $11.3(3.7$ to 35.1$)$ & $2.6(1.6$ to 4.2$)$ & $0.2(0.1$ to 0.5$)$ \\
\hline Preoperative culture & $59.4(7.3$ to 482.3$)$ & $21.5(3.1$ to 149.8$)$ & $0.4(0.2$ to 0.6$)$ \\
\hline Lenkocyte Esterase & $105.1(12.4$ to 895.0$)$ & $23.5(3.4$ to 162.7$)$ & $0.22(0.1$ to 0.4$)$ \\
\hline Leukocyte count & $42.5(6.9$ to 262.4$)$ & $7.9(2.1$ to 29.7$)$ & $0.2(0.1$ to 0.5$)$ \\
\hline Frozen section & $161.8(8.1$ to 3223.6$)$ & & $0.21(0.1$ to 0.5$)$ \\
\hline Histology & $616.3(23.7$ to 15991.3$)$ & & $0.1(0.01$ to 0.3$)$ \\
\hline
\end{tabular}

ESR/CRP: Erythrocyte Sedimentation Rate/C-Reactive Protein

CI: Confidence Interval

PJI is one of the most devastating complications of total joint arthroplasty with a relevant socioeconomic burden. More than 25\% of revisions are attributed to infections, with a reported trend to increase [19-21]. Despite new achievements in this field, PJI diagnosis remains a serious challenge and no single standard reference test has been identified to date.

Alpha defensins are a family of mammalian peptides, showing relevant microbicidal properties against many Gram-negative and Gram-positive bacteria, fungi, and enveloped viruses. They are produced, mainly by neutrophils, constitutively and/or in response to microbial products or proinflammatory cytokines. We performed a study in a Single specialized Centre for Bone Infections to evaluate the utility and possible added value of a POCT a-defensine qualitative test (Synovasure ${ }^{\mathrm{TM}}$ ) in our clinical setting.

Published data shows promising results of a-D testing in PJI with a sensitivity ranging from $63 \%$ to $100 \%[7,22-24]$ and a sensitivity of $63 \%$ on periprosthetic shoulder arthroplasty infection while in the hip and knee joints the sensitivity grows up to $97 \%$ with a specificity of $95 \%$ to $100 \%$ [25]. 
An independent study on sixty-one painful hip and knee arthroplasties or before second stage reimplantation showed a sensitivity and specificity of a-defensine testing of $100 \%$ and $95 \%$, respectively. This test demonstrated better sensitivity and specificity when compared to culture, SF leukocyte count, ESR and CRP. No data about systemic inflammatory comorbidities or metallosis was presented [26].

Recently, Synovasure ${ }^{\mathrm{TM}}$, a new POCT test for qualitative assessment of $a-D$ in the $S F$, has been developed and is now commercially available. To our knowledge, only 5 studies have been published about this new $a-D$ test qualitative showing a general decrease in diagnostic accuracy when compared to immunoassay quantitative assay [27]. The results obtained from 40 hip and knee arthroplasty revisions (12 PJI according to MSIS criteria) lead to 2 main conclusions. Firstly, Synovasure ${ }^{\mathrm{TM}}$ provide high accuracy (85\%) in PJI diagnosis, at least equivalent to intraoperative frozen section. The second conclusion confirms that, although Synovasure ${ }^{\mathrm{TM}}$ provides good results, they are not as good as previous data obtained with immunoassay laboratory test. The authors explained their false negative results with the contamination of samples with blood or cellular debris however our data, though in a limited number of cases, clearly shows that blood contamination does not influence the reliability of Synovasure ${ }^{\mathrm{TM}}$ test. In a single-centre study based on 49 patients, the authors achieved a sensitivity of $69 \%$ and a specificity of $94 \%$. a-D qualitative test achieves the same accuracy of definitive histology and one positive culture [28]. One of the added values of a POCT device use for a-D testing in this specific clinical setting undoubtedly relies on its easiness and speed of use. Some false results could be partially explained by the fact that 15 patients had an in-situ spacer at the moment of Synovasure $^{\mathrm{TM}}$ test. A further matter of debate is whether $a-D$ could be a reliable test even before a second stage revision.

In another study aimed to evaluate the accuracy of quantitative a-D test in a cohort of 102 candidates for hip or knee revision surgery. Thirty-eight of them were second-stage revisions. Although optimal accuracy was achieved in first/single-stage revisions, the sensitivity, specificity, positive and negative likelihood ratios were 67\%, 97\%, 21 and 0.3 respectively [29]. This data could be partially explained by the fact that MSIS criteria and a-D test have not been designed for second-stage revisions and by the low number of patients who may not be representative of a real setting.

High performance of a-D lateral flow test in knee PJI diagnosis was demonstrated [30]. Although their data (sensitivity $87.5 \%$ and specificity $97.1 \%$ ) are consistent with our results, the authors demonstrated the superiority of Synovasure ${ }^{\mathrm{TM}}$ in terms of sensitivity and negative predictive value when compared to other typical analyses (culture, synovial cell count, ESR and CRP).

On the other hand, recently published data based on a multicentre study of a Cohort of 121 patients was prospectively evaluated for painful hip and knee arthroplasties and screened for PJI with a-D test and MSIS criteria. In this setting, Synovasure ${ }^{\mathrm{TM}}$ showed a sensitivity, specificity, LR+ and LR- of $97.1 \%, 96.5 \%, 28.2$ and 0.03 respectively with an accuracy of $96.7 \%$. Apart from SF leukocyte count, a-D overtakes all other MSIS diagnostic test [31]. The authors do not consider the LE test in the toolbox of possible tests for PJI and preferred to retain the purulence around the prosthesis as minor criterion making a real comparison with our series difficult.

Similar data was recently reported utilizing the largest a-D cohort published up until now [32]. 191 patients (195 joints) were screened for MSIS criteria and included in this prospective study. Synovasure ${ }^{\mathrm{TM}}$ test was performed on all patients and when possible, a quantitative ELISA test was carried out (173 patients). Sensitivity, specificity, PPV and NPV of Synovasure $^{\mathrm{TM}}$ test were $92.1 \%, 100 \%, 100 \%$ and $95.2 \%$, respectively. The accuracy was $96.9 \%$. Interestingly, the accuracy of the lateral flow analysis was $94.8 \%$ when compared to the laboratory immunoassay.

According to our data, Synovasure ${ }^{\mathrm{TM}}$ test demonstrated statistical significant difference only when compared to 1 positive culture sensitivity and ESR/CRP specificity. Though these results, we were able to screen the whole cohort of patients only for a-D, blood test and culture analysis. LE was performed on $67(91.8 \%)$ out of 73 patients due to blood contamination or lack of SF. This finding is in line with previous presented data [23]. They pointed out that the LE test was unreadable in eight of 46 joints $(17 \%)$ as a result of blood interference. Small volumes of retrieved SF limited leukocyte count and neutrophil percentage analysis (43 and 15 patients respectively). Moreover, intraoperative criteria such as frozen section and histology were available only in 42 and 43 patients, respectively. This data clearly shows that a Synovasure ${ }^{\mathrm{TM}}$ test outperforms the majority of MSIS criteria in the number of potentially screenable patients.

Some authors tried to improve the effectiveness of a-D in PJI diagnosis combining it with other serum or SF analysis [22] testing the combination of a-D and serum CRP dosage in 149 patients affected by painful arthroplasty, 37 of them were later diagnosed by PJI 
according to MSIS criteria. While the only a-D dosage demonstrated a specificity of $95.5 \%$ and a sensitivity of $97.3 \%$, the combination of both tests resulted in a specificity of $100 \%$ and a sensitivity of $99.3 \%$. Synovial CRP reversed all-false positive $\alpha-D$ data and lead to a correct diagnosis in $99 \%$ of patients.

Combining our a-D results with LE data, Synovasure $^{\mathrm{TM}}$ correctly reversed the only false positive tests obtained with LE. Out of the 8 false negatives LE tests, only 4 were diagnosed by a-D leading to a combined sensitivity of $88.2 \%$ (95\% CI 72.6 to 96.7$)$ and specificity of $100 \%$ (95\% CI 89.4 to 100).

The Alpha-defensin test already shows its reliability regardless preceding antibiotic treatment [33]. Our data is consistent with this evidence and demonstrated that Synovasure ${ }^{\mathrm{TM}}$ is a reliable test even in presence of blood contamination of SF samples. The relatively high percentage $(13.4 \%)$ of patients under antibiotic therapy started before referral to a specialized hospital, highlight the need of educational improvement of primary care providers who deal with patients with painful joint arthroplasty [34].

Undoubtedly, our study has some limitations. Point of care test seems to provide inferior results especially for sensitivity values when compared to quantitative immunoassay test, however we cannot directly compare our data with previously published evidence obtained with laboratory assays. Moreover, we did not succeed to screen all patients for each individual test. This limitation could be bias to the data analysis. The study lacks statistical power preventing us to draw firm conclusions. A final weakness is that we included patients under antibiotic therapy that could influence SF leukocyte count, neutrophil percentage, and culture and therefore compromise the diagnosis of PJI [35]. Nevertheless, the authors aimed to report a practical everyday life experience of a bone infection unit in which antibiotic therapy at the moment of SF aspiration and blood sample contamination are common situations.

\section{Conclusion}

Despite the limitations discussed above, our findings show that Synovasure ${ }^{\mathrm{TM}}$ sensitivity is lower than quantitative a-D test, but it is a reliable and easy-to-use option for PJI diagnosis when compared to current MSIS criteria. Synovasure ${ }^{\mathrm{TM}}$, compared to the main available tests, shows good specificity and the highest DOR. On the SF it is the easier test to do, due to the fact that it needs a minimal amount of SF and it is not limited by blood contamination or antibiotic use.
Whereas there is no single stand alone test, we believe that Synovasure ${ }^{\mathrm{TM}}$ holds the potential to be included in the toolbox of tests for PJI diagnosis.

\section{Abbreviations}

PJI: periprosthetic joint infection; MSIS: the Musculoskeletal Infection Society; THA: total hip arthroplasty; TKA: total knee arthroplasty; ESR: erythrocyte sedimentation rate; CRP: C-reactive protein; WBC: white blood cell; LE: leukocyte esterase; SF: synovial fluid; RSA: shoulder arthroplasties; RA: rheumatoid arthritis; MRSA: methicillin-resistant staphylococcus aureus; LR+: positive likelihood ratio; LR-: negative likelihood ratio; DOR: Diagnostic Odds ratio; CI: Confidence Intervals.

\section{Acknowledgments}

We acknowledge the Clinical Trials Centre of the Ospedale Policlinico San Martino - University of Genoa (Italy) for the statistical analysis.

\section{Competing Interests}

The authors have declared that no competing interest exists.

\section{References}

1. Kurtz SM, Lau E, Watson H, Schmier JK, Parvizi J. Economic burden of periprosthetic joint infection in the United States. J Arthroplasty. 2012 Sep;27(8 Suppl):61-5.e1. doi: 10.1016/j.arth.2012.02.022. Epub 2012 May 2.

2. Carrega G, Bartolacci V, Burastero G, Casalino Finocchio G, Grappiolo G, Ronca A, Salomone C, Riccio G. Surgery-related infections following arthoplasty: a prospective survey in a tertiary-care center. Minerva Ortopedica e Traumatologica 2011 February;62(1):1-8

3. Ulrich SD, Seyler TM, Bennett D, Delanois RE, Saleh KJ, Thongtrangan I, Kuskowski M, Cheng EY, Sharkey PF, Parvizi J, Stiehl JB, Mont MA. Total hip arthroplasties: what are the reasons for revision? Int Orthop. 2008 Oct;32(5):597-604. Epub 2007 Apr 19.

4. Koh CK, Zeng I, Ravi S, Zhu M, Vince KG, Young SW. Periprosthetic Joint Infection Is the Main Cause of Failure for Modern Knee Arthroplasty: An Analysis of 11,134 Knees. Clin Orthop Relat Res. 2017 Sep;475(9):2194-2201. doi: 10.1007/s11999-017-5396-4. Epub 2017 Jun 1.

5. Parvizi J, Zmistowski B, Berbari EF, et al. New definition for periprosthetic joint infection: from the Workgroup of the Musculoskeletal Infection Society. Clin Orthop Relat Res 2011;469:2992-2994.

6. Parvizi J, Gehrke T, Chen AF. Proceedings of the International Consensus on Periprosthetic Joint Infection. Bone Joint J 2013;95-B:1450-1452.

7. Deirmengian C, Kardos K, Kilmartin P, Cameron A, Schiller K, Parvizi J. Diagnosing periprosthetic joint infection: has the era of the biomarker arrived? Clin Orthop Relat Res. 2014 Nov;472(11):3254-62. doi: 10.1007/s11999-014-3543-8

8. Ganz T, Selsted ME, Szklarek D, Harwig SS, Daher K, Bainton DF, Lehrer RI. Defensins Natural peptide. J Clin Invest. 1985 Oct;76(4):1427-35.

9. Lehrer RI, Ganz T. Defensis: endogenous antibiotic peptides from human leukocytes. Ciba Found Symp. 1992;171:276-90; discussion 290-3

10. Wyatt MC, Beswick AD, Kunutsor SK, Wilson MJ, Whitehouse MR, Blom AW The Alpha-Defensin Immunoassay and Leukocyte Esterase Colorimetric Strip Test for the Diagnosis of Periprosthetic Infection: A Systematic Review and Meta-Analysis. J Bone Joint Surg Am. 2016 Jun 15;98(12):992-1000. doi: 10.2106/JBJS.15.01142.

11. Pupaibool J, Fulnecky EJ, Swords RL Jr, Sistrunk WW, Haddow AD. Alpha-defensin-novel synovial fluid biomarker for the diagnosis of periprosthetic joint infection. Int Orthop. 2016 Dec;40(12):2447-2452. Epub 2016 Oct 7.

12. Li B, Chen F, Liu Y, Xu G. Synovial Fluid a-Defensin as a Biomarker for Peri-Prosthetic Joint Infection: A Systematic Review and Meta-Analysis. Surg Infect. (Larchmt) 2017 Aug/Sep;18(6):702-710. doi: 10.1089/sur.2017.006.

13. Xie K, Qu X, Yan M. Procalcitonin and a-Defensin for Diagnosis of Periprosthetic Joint Infections. J Arthroplasty.2017 Apr;32(4):1387-1394. doi:10.1016/j.arth.2016.10.001. 
14. Yuan J, Yan Y, Zhang J, Wang B, Feng J. Diagnostic accuracy of alpha-defensin in periprosthetic joint infection: a systematic review and meta-analysis. Int Orthop. 2017 Dec;41(12):2447-2455. doi: 10.1007/s00264-017-3647-3.

15. Suen K, Keeka M, Ailabouni R, Tran P. Synovasure 'quick test' is not as accurate as the laboratory-based a-defensin immunoassy: a systematic review and meta-analysis. Bone Joint J. 2018 Jan;100-B(1):66-72. doi: 10.1302/0301-620X.100B1.BJJ-2017-0630.R1.

16. Parvizi J, Gehrke T. Definition of periprosthetic joint infection. J Arthroplasty.2014 Jul;29(7):1331. doi: 10.1016/j.arth.2014.03.009.

17. Colvin OC, Kransdorf MJ, Roberts CC, et al. Leukocyte esterase analysis in the diagnosis of joint infection: can we make a diagnosis using a simple urine dipstick? Skeletal Radiol 2015;44(5):673.

18. Zimmerli W, Trampuz A, Ochsner PE. Prosthetic-Joint Infections. N Engl J Med 2004; 351:1645-1654.

19. Kurtz S, Ong K, Lau E, Mowat F, Halpern M. Projections of primary and revision hip and knee arthroplasty in the United States from 2005 to 2030. J Bone Joint Surg Am 2007; 89: 780-85.

20. Bozic KJ, Kurtz SM, Lau E, et al. The epidemiology of revision total knee arthroplasty in the United States. Clin Orthop Relat Res 2010; 468: 45-51.

21. Burastero G, Basso M, Carrega G, Cavagnaro L, Chiarlone F, Salomone C, Papa G, Felli L. Acetabular spacers in 2-stage hip revision: is it worth it? A single-centre retrospective study. Hip Int.2017 Mar 31;27(2):187-192. doi: 10.5301/hipint.5000446.

22. Deirmengian $C$, Kardos $K$, Kilmartin $P$, Cameron A, Schiller $K$, Parvizi J. Combined measurement of synovial fluid a-Defensin and C-reactive protein levels: highly accurate for diagnosing periprosthetic joint infection. J Bone Joint Surg Am. 2014 Sep 3;96(17):1439-45. doi: 10.2106/JBJS.M.01316.

23. Deirmengian C, Kardos K, Kilmartin P, Cameron A, Schiller K, Booth RE Jr, Parvizi J. The alpha-defensin test for periprosthetic joint infection outperforms the leukocyte esterase test strip. Clin Orthop Relat Res. 2015 Jan;473(1):198-203. doi: 10.1007/s11999-014-3722-7.

24. Bonanzinga T, Zahar A, Dütsch M, Lausmann C, Kendoff D, Gehrke T. How Reliable Is the Alpha-defensin Immunoassay Test for Diagnosing Periprosthetic Joint Infection? A Prospective Study. Clin Orthop Relat Res. 2017 Feb;475(2):408-415. doi: 10.1007/s11999-016-4906-0.

25. Frangiamore SJ, Saleh A, Grosso MJ, Kovac MF, Higuera CA, Iannotti JP, Ricchetti ET. A-Defensin as a predictor of periprosthetic shoulder infection. J Shoulder Elbow Surg.2015 Jul;24(7):1021-7. doi: 10.1016/j.jse.2014.12.021.

26. Bingham J, Clarke H, Spangehl M, Schwartz A, Beauchamp C, Goldberg B. The alpha defensin-1 biomarker assay can be used to evaluate the potentially infected total joint arthroplasty. Clin Orthop Relat Res. 2014 Dec;472(12):4006-9. doi: 10.1007/s11999-014-3900-7.

27. Kasparek MF, Kasparek M, Boettner F, Faschingbauer M, Hahne J, Dominkus M. Intraoperative Diagnosis of Periprosthetic Joint Infection Using a Novel Alpha-Defensin Lateral Flow Assay. J Arthroplasty. 2016 Dec;31(12):2871-2874. doi:10.1016/j.arth.2016.05.033.

28. Sigmund IK, Holinka J, Gamper J, Staats K, Böhler C, Kubista B, Windhager R. Qualitative a-defensin test (Synovasure) for the diagnosis of periprosthetic infection in revision total joint arthroplasty. Bone Joint J. 2017 Jan;99-B(1):66-72. doi: 10.1302/0301-620X.99B1.BJJ-2016-0295.R1.

29. Frangiamore SJ, Gajewski ND, Saleh A, Farias-Kovac M, Barsoum WK, Higuera CA. a-Defensin Accuracy to Diagnose Periprosthetic Joint Infection-Best Available Test? J Arthroplasty. 2016 Feb;31(2):456-60. doi: 10.1016/j.arth.2015.09.035.

30. Balato $G$, Franceschini $V$, Ascione $T$, Lamberti A, D'Amato $M$, Ensini $A$, Baldini A. High performance of a-defensin lateral flow assay (Synovasure) in the diagnosis of chronic knee prosthetic infections. Knee Surg Sports Traumatol Arthrosc. 2017 Oct 7. doi:10.1007/s00167-017-4745-x.

31. Berger P, Van Cauter M, Driesen R, Neyt J, Cornu O, Bellemans J. Diagnosis og prosthetic joint infection with alph-defensin using a lateral flow device: a multicentre study. Bone Joint J. 2017 Sep;99-B(9):1176-1182. doi: 10.1302/0301-620X.99B9.BJJ-2016-1345.R2.

32. Gehrke T, Lausmann C, Citak M, Bonanzinga T, Frommelt L, Zahar A. The Accuracy of the Alpha Defensin Lateral Flow Device for Diagnosis of Periprosthetic Joint Infection: Comparison with a Gold Standard. Jbone Joint SurgAm.2018 Jan 3;100(1):42-48. doi: 10.2106/JBJS.16.01522.

33. Shahi A, Parvizi J, Kazarian GS, Higuera C, Frangiamore S, Bingham J, Beauchamp C, Valle CD, Deirmengian C. The Alpha-defensin Test for Periprosthetic Joint Infections Is Not Affected by Prior Antibiotic Administration. Clin Orthop Relat Res.2016 Jul;474(7):1610-5. doi: 10.1007/s11999-016-4726-2.

34. Spangehl MJ. CORR Insights: The Alpha-defensin Test for Periprosthetic Joint Infection is Not Affected by Prior Antibiotic Administration. Clin Orthop Relat Res 2016 Jul;474(7):1616-8. doi: 10.1007/s11999-016-4771-x.

35. Shahi A, Deirmengian C, Higuera C, Chen A, Restrepo C, Zmistowski B, Parvizi J. Premature Therapeutic Antimicrobial Treatments Can Compromise the Diagnosis of Late Periprosthetic Joint Infection. Clin Orthop Relat Res. 2015 Jul;473(7):2244-9. doi: 10.1007/s11999-015-4142-z. 